\title{
Role of low molecular weight heparin and low dose aspirin in recurrent pregnancy loss with APLA /pre APLA syndrome and hyperhomocysteinemia: our experience
}

\author{
Madhuri R Dabade, Rajiv T Dabade, Avdhut Kashid, Sadhana P Deshmukh, \\ Pradeep M Patil, Suman P Sardesai
}

\begin{abstract}
Corresponding author: Dr. Rajiv T Dabade, Associate Professor, Department of Obstetrics and Gynecology, Ashwini Rural Medical College, Hospital and Research Centre, Kumbhari, Solapur, Maharashtra, India; Email : ddguru2019@gmail.com
\end{abstract}

Distributed under Attribution-Non Commercial - Share Alike 4.0 International (CC BY-NC-SA 4.0)

\begin{abstract}
Objective: The purpose of the study was to see the effect of low molecular weight heparin (LMWH) and low dose aspirin (LDA) in recurrent pregnancy losses (RPL) due to antiphospholipid antibody (APLA) / Pre APLA syndrome and hyperhomocysteinemia. Method: A total number of 138 women presenting in pregnancy with previous two or more consecutive pregnancy losses with APLA / Pre APLA syndrome or with hyperhomocysteinemia (HHCY) were included in the study. APLA tests and serum homocysteine levels were done in all cases. Patients were put on LMWH $40 \mathrm{mg} \mathrm{sc}$ /day and tab aspirin $150 \mathrm{mg}$ /day after the cardiac activity was noted and continued till twelve hours before planned delivery. In cases of hyperhomocysteinemia vitamin B12 and folic acid supplementation was given along with thromboprophylaxis. Results: Out of 138 women with RPL, 105 (76.07\%) had recurrent abortions while 52 $(37.68 \%)$ had history of intrauterine deaths in past pregnancy along with obstetric complications like preeclampsia, IUGR etc. APLA test was positive in 42 women $(30.43 \%)$ while it was negative in $86(67.18 \%)$ women. Hyperhomocysteinemia was present in $10(7.24 \%)$ women. Women who received thromboprophylaxis the outcome in index pregnancy was, $94.20 \%$ i.e. 130 women had successful pregnancy outcome (live births) while 8 women (6\%) had pregnancy loss in spite of thromboprophylaxis and vitamin B12 supplementation. Conclusion: Combination treatment with LDA and LMWH in women with APLA / Pre APLA syndrome and additional vitamin B12 and folic acid supplementation in hyperhomocysteinemia significantly improves the pregnancy outcome in patients with recurrent pregnancy loss with success rate of $94 \%$.
\end{abstract}

Keywords: APLA syndrome, pre APLA syndrome, low molecular weight heparin, low dose aspirin. thromboprophylaxis, hyperhomocysteinemia (HHCY).

Recurrent Pregnancy loss (RPL) is s distressing event for both the woman and her family. It is a challenging situation for the treating obstetrician to evaluate the woman with RPL and prevent recurrence in present pregnancy. One of the causes of RPL is antiphospholipid antibody syndrome (APLA) and hyperhomocysteinemia (HHCY). The protocols for management of APLA syndrome are rapidly changing, and this causes confusion in the minds of treating obstetrician. Few years back low molecular weight heparin
(LMWH) was the main stay of treatment of APLA syndrome but in recent years it has not been recommended. We all agree that it should be used judiciously; on the other hand our experience does support the use of LMWH and low dose aspirin (LDA) in selected cases of recurrent pregnancy loss. Hyperhomocysteinemia has also emerged as a strong risk factor for RPL and it has a correlation with complications such as spontaneous abortion fetal death, preeclampsia and placental abruption etc. ${ }^{1}$ LMWH in combination with low

Received: $29^{\text {th }}$ December 2020, Peer review completed: $27^{\text {th }}$ February 2021, Accepted: $7^{\text {th }}$ March 2021.

Dabade MR, Dabade RT, Kashid A, Deshmukh SP, Patil PM, Sardesai SP. Role of low molecular weight heparin and low dose aspirin in recurrent pregnancy loss with APLA /pre APLA syndrome and hyperhomocysteinemia: our experience. The New Indian Journal of OBGYN. 2022; 8(2): 200 - 203. 
dose aspirin has shown improvement in live birth rate and decline in associated morbidities like preeclampsia eclampsia, intrauterine growth restriction (IUGR) and prematurity in patients with APLA syndrome. ${ }^{2}$ Vitamin B12 $3000 \mathrm{mg}$ and folic acid supplementation in patients with hyperhomocysteinemia along with thromboprophylaxis also gives good obstetric outcome. ${ }^{3}$

Many women of RPL with history and associated morbidities are very suggestive of APLA syndrome but they are APLA negative on investigation. There is an entity called probable APLA / Pre APLA syndrome. In these cases without thromboprophylaxis the miscarriage rate in subsequent pregnancy is as high as $90 \% .{ }^{4}$ Keeping in mind the above fact the present study was under taken to assess the effect of thromboprophylaxis with LMWH and LDA in woman with RPL with APLA positive or history very suggestive of APLA syndrome but APLA negative i.e. Pre APLA syndrome. In women with hyperhomocysteinemia additional vitamin B12 supplementation, folic acid along with thromboprophylaxis was given. The outcome of pregnancy along with associated morbidities was assessed in index pregnancy.

\section{Material and methods}

The present prospective study included 138 women with history of two or more recurrent pregnancy losses with confirmed or suggestive of APLA syndrome or with HHCY was carried out at Krishnamai hospital and Ashwini Sahakari Rugnalaya over a period of 5 years from 2015 to 2019. Other causes of pregnancy losses like anatomical abnormality, luteal phase defect, infections etc were excluded.

Detail history, about previous obstetric complications was obtained and baseline routine investigations were done. In view of APLA syndrome, lupus anticoagulant, anticardiolipin antibody $\mathrm{IgG}$, anti B2 glycoprotein $\operatorname{IgA}$, antiphospholipid estimation was done. Serum homocysteine was done in all cases.

Therapy in the form of low dose aspirin (LDA) $150 \mathrm{mg}$ and injection enoxaparine (LMWH) 40mg per day was commenced once fetal viability was detected on ultrasonography (USG) and continued till 36 weeks. Coagulation profile PT-INR was done every month till patient was on Inj. enoxaparine. In cases of HHCY vitamin B12 (cynocobalamine) was given in the dose of $3000 \mathrm{mg}$ and folic acid $5 \mathrm{mg}$ per day. There are no references available regarding the use of LMWH and LDA or vitamin B12 alone in cases of RPL with hyperhomocysteinemia. The basic pathology of HHCY is endothelial cell injury, platelet aggregation and formation of microthrombi very similar to
APLAS and hence we treated these patients with thromboprophylaxis like LMWH + LDA as in APLA syndrome along with cynocobalamine and folic acid.

Routine prenatal care was done to monitor hypertension, proteinuria and periodic USG to assess the fetal growth, amniotic fluid volume, doppler flow studies etc. Decision regarding induction or caesarean section was taken depending on the presence or absence of associated obstetric complications and fetal condition after 37 weeks of gestation or earlier if needed. Coagulation profile was done every month. Primary outcome measure was the rate of live births, and secondary outcome included obstetrical complications like pre-eclampsia, eclampsia, HELLP syndrome, IUGR, abruption placenta etc. Neonatal events were evaluated after delivery.

Time of testing for APLA: APLA may appear transiently in normal individuals hence it is recommended that APLA assay is of diagnostic value if performed during pregnancy or at a time close to pregnancy loss. Serum homocysteine estimation is done with 14 hours of starvation and $10.5 \mathrm{umol}$ is taken as cutoff in pregnancy.

Statistical analysis: Descriptive statistics such as mean, standard deviation (SD) and percentage was used to present the data. Associated obstetric complications in past pregnancies and in index pregnancies were assessed by using chi-square test. A p-value less than 0.05 was considered as significant. Data analysis was performed by using software SPSS version 20.0.

\section{Results}

Majority of patients were belongs to age group 25-29 (43.47\%) followed by $20-24$ (42.75\%) (table 1$)$.

\begin{tabular}{lll}
\multicolumn{2}{l}{ Table 1: Age wise distribution } & \\
\hline Age & No. of cases & Percentage (\%) \\
\hline $15-19$ & 1 & 0.72 \\
$20-24$ & 59 & 42.75 \\
$25-29$ & 60 & 43.47 \\
$30-34$ & 18 & 13.04 \\
\hline
\end{tabular}

Late fetal loss i.e. intrauterine foetal death (IUFD) is strongly associated with presence of antiphospholipid antibodies particularly lupus anticoagulant. $2.89 \%$ of the patients had preterm deliveries with neonatal deaths (table 2). Preterm induction was needed in patients with severe preeclampsia / eclampsia or in pregnancy with severe IUGR. Increased incidence of preterm deliveries can be attributed to both the disease process and as iatrogenic factor because of preterm inductions.

Lupus antibody is the most powerful predictor of thrombosis and recurrent miscarriages. Anti beta2 glycoprotein antibodies are not associated with recurrent 
The New Indian Journal of OBGYN. 2021 (January-June);8(2)

miscarriages in isolation however in combination with positive results for lupus anticoagulant (LA) and anticardiolipin antibody (ACL), there is high risk of obstetric complications. $66 \%$ patients were APLA negative and they might be cases of pre APLA or seronegative APLA syndrome (table 3 ).

\begin{tabular}{lll} 
Table 2: Previous pregnancy losses & & \\
\hline Previous pregnancy loss & $\begin{array}{l}\text { No. of } \\
\text { cases }\end{array}$ & $\begin{array}{l}\text { Percentage } \\
(\%)\end{array}$ \\
\hline Abortion - 1 & 21 & 15.21 \\
Abortion - 2 & 51 & 36.95 \\
Abortion - 3 & 33 & 23.91 \\
Intrauterine foetal death (IUFD) & 52 & 37.68 \\
Preterm deliveries with neonatal deaths & 4 & 2.89 \\
\hline Two pregnancy losses were of one type or combinations of events \\
without a live pregnancy in between were included in the study.
\end{tabular}

\begin{tabular}{lll} 
Table 3: APLA tests & & \\
\hline APLA / Homocysteine & $\begin{array}{l}\text { No. of } \\
\text { patients }\end{array}$ & $\begin{array}{l}\text { Percentage } \\
\mathbf{( \% )}\end{array}$ \\
\hline Lupus anticoagulant & 17 & 12.31 \\
Antiphospholipid antibody & 13 & 9.45 \\
Anticardiolipin antibody & 9 & 6.52 \\
APLA negative & 86 & 67.18 \\
Serum homocysteine & 10 & 7.24 \\
\hline
\end{tabular}

Table 4: Incidence associated obstetric complications in past pregnancies and in index pregnancies

\begin{tabular}{|c|c|c|c|c|}
\hline \multirow{2}{*}{$\begin{array}{l}\text { Categories } \\
\text { Associated } \\
\text { obstetric } \\
\text { complications }\end{array}$} & \multicolumn{2}{|c|}{ Past pregnancy } & \multicolumn{2}{|c|}{ Index pregnancy } \\
\hline & $\begin{array}{l}\text { No of } \\
\text { cases }\end{array}$ & $\begin{array}{l}\text { Percentage } \\
(\%)\end{array}$ & $\begin{array}{l}\text { No of } \\
\text { cases }\end{array}$ & $\begin{array}{l}\text { Percentage } \\
(\%)\end{array}$ \\
\hline Pre-eclampsia & 16 & 11.59 & 7 & 5.07 \\
\hline Eclampsia & 4 & 2.89 & 1 & 0.72 \\
\hline IUGR & 7 & 5.07 & 3 & 2.17 \\
\hline Oligohydramnios & 7 & 5.07 & 6 & 4.34 \\
\hline Abruptio-placentae & 2 & 1.44 & 2 & 1.44 \\
\hline $\mathrm{PPH}$ & 0 & 0 & 1 & 0.72 \\
\hline
\end{tabular}

$34(24.63 \%)$ women out of 138 women had significant associated obstetric complications in previous pregnancies as per history and preeclampsia - eclampsia, being the commonest complication $(p<0.05)$. Early onset severe preeclampsia with HELLP syndrome is a frequent association. About one third untreated patients with APLA syndrome may develop preeclampsia during pregnancy and more than $10 \%$ will deliver small for gestational age infants. The incidence of obstetric complications was less in index pregnancy probably because of thromboprophylaxis. The successful pregnancy outcome was noted in $94 \%$ of the patients while $6 \%$ had pregnancy loss in index pregnancy in spite of thromboprophylaxis. Out of 8 losses 6 cases were APLA positive while one was APLA negative and one case was of hyperhomocysteinemia.

\section{Discussion}

Adverse pregnancy outcome in woman with APLA syndrome is poor placental perfusion due to localized thrombosis. Hyperhomocysteinemia also causes hypercoagulable state, thrombosis and adverse pregnancy outcome very similar to APLA syndrome. The treatment of APLA or pre APLA syndrome is thromboprophylaxis in the form of LMWH and LDA. It is still unclear what treatment is required for women who do not meet all the criteria for diagnosis of APLA but have all clinical features of APLA syndrome. A positive approach towards this condition is suggested.

According to cochrane study 2014, low dose aspirin alone was found to be inferior to LDA and LMWH ${ }^{5}$. This combination may promote successful embryonic implantation in the early stages of pregnancy and protect against thrombosis of the vasculature after successful implantation and decrease incidence of IUGR and PIH. This therapy also helps in reducing obstetric morbidity. In our study the success rate in APLA / Pre APLA with thromboprophylaxis was $94 \%$, which is very satisfying. Humade reported success rate of $87 \%$ in cases of recurrent miscarriage in patients with hyperhomocysteinemia ${ }^{6}$. They were treated with vitamin B6, B12 3000mg before pregnancy and continued during pregnancy. In our study, out of 10 cases of hyperhomocysteinemia, 9 (90\%) had successful pregnancy outcome with vitamin $\mathrm{B} 12$ and folic acid supplementation along with LMWH and LDA.

Randomized controlled trials will only guide us whether there is role of thromboprophylaxis or not in APLA / Pre APLA syndrome and in hyperhomocysteinemia. Till then we should give these women a benefit of having a live baby and reduce the obstetric complication associated with it by thromboprophylaxis.

\section{Conclusion}

Combination treatment with aspirin and LMWH in patients with APLA / pre APLA and additional vitamin B12 supplementation in hyperhomocysteinemia can significantly improve live birth rates up to more than $90 \%$. Obstetric complications such as preeclampsia, fetal growth restriction and prematurity remain high despite of treatment, but the severity is less. Close antenatal surveillance and planned delivery of these pregnancies in a unit with specialist obstetric and neonatal intensive care facilities are indicated.

\section{Conflict of interest: None. Disclaimer: Nil.}


The New Indian Journal of OBGYN. 2021 (January-June);8(2)

\section{References}

1. Gaiday AN, Tussupkaliyev AB, Bermagambetova SK, Zhumagulova SS, Sarsembayeva LK, Dossimbetova $\mathrm{MB}$, et al. Effect of homocysteine on pregnancy: A Systematic Review Chemico Bilological Interaction. 2018; 293: $70-6$.

2. de Jesus GR, Rodrigues G, de Jesús NR. Pregnancy Morbidity, in Antiphospholipid Syndrome: what is the impact of treatment ?. Curr Rheumatol Rep. 2014; 16: 403

3. Mukhopadhyay I, Pruthviraj V, Rao PS, Biswas M. Hyperhomocysteinemia in recurrent pregnancy loss and the effect of folic acid and vitamin B12 on homocysteine levels: a prospective analysis. International Journal of Reproduction, Contraception, Obstetrics and Gynecology. 2017; 6(6): 2258 - 61 .

4. Abd Aziz Mohammad K, Sanny Saad A. Enoxaparine and aspirin therapy for recurrent pregnancy loss due to antiphospholipids syndrome (APS). Middle East Fertility Society Journal. 2014; 19:176 - 82

5. de Jong PG, Kaandorp S, Nisio MD, Goddijn M, Middeldorp S, Cochrane Pregnancy and Childbirth Group. Aspirin and / or Heparin for women with unexplained recurrent miscarriage with or without inherited Thrombophilia. Cochrane Database Syst Rev. 2014 Jul; 2014(7): CD004734
6. Humadi E. Treatment of Hyperhomocysteinemia and Pregnancy outcome in patients with recurrent miscarriage. Mustansiriya Medical Journal. 2016; 15(2); $9-13$.

Madhuri R Dabade ${ }^{1}$, Rajiv T Dabade ${ }^{2}$, Avdhut Kashid ${ }^{3}$, Sadhana P Deshmukh ${ }^{4}$, Pradeep M Patil ${ }^{5}$, Suman P Sardesai ${ }^{6}$

${ }^{1}$ Consultant in OBGY at Krishnamai Hospital and Ashwini Sahakari Rugnalaya ANI Sanshodhan Kendra, Solapur, Maharashtra, India; ${ }^{2}$ Associate Professor, Department of Obstetrics and Gynecology, Ashwini Rural Medical College, Hospital and Research Centre, Kumbhari, Solapur, Maharashtra, India; ${ }^{3}$ Department of Obstetrics and Gynecology, Ashwini Rural Medical College, Hospital and Research Centre, Kumbhari, Solapur, Maharashtra, India; ${ }^{4}$ Department of Obstetrics and Gynecology, Ashwini Rural Medical College, Hospital and Research Centre, Kumbhari, Solapur, Maharashtra, India; ${ }^{5}$ Department of Obstetrics and Gynecology, Ashwini Rural Medical College, Hospital and Research Centre, Kumbhari, Solapur, Maharashtra, India; ${ }^{6}$ Department of Obstetrics and Gynecology, Ashwini Rural Medical College, Hospital and Research Centre, Kumbhari, Solapur, Maharashtra, India. 